\title{
PCR amplification of Bartonella koehlerae from human blood and enrichment blood cultures
}

\author{
Edward B Breitschwerdt ${ }^{1 *}$, Ricardo G Maggi', B Robert Mozayeni², Barbara C Hegarty ${ }^{1}$, Julie M Bradley ${ }^{1}$, \\ Patricia E Mascarelli ${ }^{1}$
}

\begin{abstract}
Background: Cats appear to be the primary reservoir host for Bartonella koehlerae, an alpha Proteobacteria that is most likely transmitted among cat populations by fleas (Ctenocephalides felis). Bartonella koehlerae has caused endocarditis in a dog and in one human patient from Israel, but other clinically relevant reports involving this bacterium are lacking. Despite publication of numerous, worldwide epidemiological studies designed to determine the prevalence of Bartonella spp. bacteremia in cats, B. koehlerae has never been isolated using conventional blood agar plates. To date, successful isolation of $B$. koehlerae from cats and from the one human endocarditis patient has consistently required the use of chocolate agar plates.

Results: In this study, Bartonella koehlerae bacteremia was documented in eight immunocompetent patients by PCR amplification and DNA sequencing, either prior to or after enrichment blood culture using Bartonella alpha Proteobacteria growth medium. Presenting symptoms most often included fatigue, insomnia, joint pain, headache, memory loss, and muscle pain. Four patients were also infected with Bartonella vinsonii subsp. berkhoffii genotype II. After molecular documentation of B. koehlerae infection in these patients, a serological test was developed and serum samples were tested retrospectively. Bartonella koehlerae antibodies were not detected (titers $<1: 16$ ) in 30 healthy human control sera, whereas five of eight patient samples had B. koehlerae antibody titers of 1:64 or greater.

Conclusions: Although biased by a study population consisting of individuals with extensive arthropod and animal exposure, the results of this study suggest that $B$. koehlerae bacteremia is more common in immunocompetent people than has been previously suspected. Future studies should more thoroughly define modes of transmission and risk factors for acquiring infection with $B$. koehlerae. In addition, studies are needed to determine if $B$. koehlerae is a cause or cofactor in the development of arthritis, peripheral neuropathies or tachyarrhythmias in patients.
\end{abstract}

\section{Background}

Bartonella koehlerae has a relative short microbiological history. In 1994, during a study designed to investigate the prevalence of Bartonella henselae bacteremia in domestic cats, B. koehlerae was isolated for the first time from the blood of two flea-infested healthy cats located on a farm in northern California [1,2]. Following experimental subcutaneous inoculation of one of these California $B$. koehlerae isolates, four cats became bacteremic for a mean of 74 days and each cat developed a

\footnotetext{
* Correspondence: ed_breitschwerdt@ncsu.edu

'Intracellular Pathogens Research Laboratory and the Center for Comparative Medicine and Translational Research, College of Veterinary Medicine, North

Carolina State University, Raleigh, NC, USA

Full list of author information is available at the end of the article
}

species-specific antibody response to B. koehlerae outer membrane proteins [3]. Subsequently, B. koehlerae DNA was amplified from cat fleas (Ctenocephalides felis) collected from pets located throughout France [4]. Eightyone of 309 fleas tested by polymerase chain reaction (PCR) and DNA sequencing contained a Bartonella spp.; B. clarridgeiae was found in $68 \%, B$. quintana in $17 \%$, B. henselae in $11 \%$, and B. koehlerae in $4 \%$. Bartonella koehlerae DNA was also amplified from an unidentified flea species removed from gerbils (Meriones lybicus) in Afghanistan [5]. Bartonella koehlerae was next isolated from a kitten in France suspected of having caused cat scratch disease in the owner [6]. Based upon these observations, cats are likely a primary reservoir host for B. koehlerae, as has been documented for

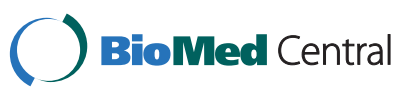


B. henselae and B. clarridgeiae, with transmission among cats most likely occurring by infestations of Ctenocephalides felis; however, neither reservoir potential nor the mode of transmission have been definitively confirmed. To date, B. koehlerae has only been reported as a human pathogen in a single patient from Israel, who was diagnosed with culture-negative aortic valve endocarditis [7]. Those investigators subsequently isolated $B$. koehlerae from stray cats in Israel, which were the presumed source of infection for their patient. In 2010, $B$. koehlerae endocarditis was reported in a dog from Israel [8].

Historically, B. henselae and B. clarridgeiae have been frequently isolated from cat blood; however, successful isolation generally required prolonged incubation (weeks) in a high $\mathrm{CO}_{2}$ incubator. Using the same isolation approaches, $B$. koehlerae has been infrequently isolated, despite numerous, worldwide epidemiological studies designed to determine the prevalence of Bartonella spp. bacteremia in cats [9]. Therefore, it appears that $B$. koehlerae is more fastidious than B. henselae or B. clarridgeiae. To date, successful isolation of B. koehlerae from cats and the one human patient has consistently required the use of chocolate agar plates $[1,2,7]$. In recent years, our research group has focused on the enhanced diagnostic detection of Bartonella spp. in healthy and sick animals and in immunocompetent human patients [10-15]. Initial efforts to enhance the sensitivity of PCR detection of Bartonella-specific DNA sequences in patient samples, as a sole molecular diagnostic strategy, proved unsatisfactory. Therefore, we incorporated pre-enrichment culture of asepticallyobtained diagnostic specimens (blood, cerebrospinal, aqueous, and joint fluids and effusions) using a liquid insect cell culture-based medium (Bartonella alpha Proteobacteria Growth Medium, BAPGM) prior to PCR testing [10-16]. By combining enrichment culture followed by PCR amplification, diagnostic sensitivity was improved substantially over PCR alone, particularly when testing samples from sick dogs and immunocompetent human patients. The use of BAPGM has also facilitated the documentation of human infections with two novel Bartonella species [14,15].

In this study, we report the molecular detection of $B$. koehlerae DNA prior to or after enrichment culture in eight patients who were tested because of a spectrum of poorly defined illnesses. After molecular documentation of $B$. koehlerae infection in these patients, a serological test was developed and serum samples were tested retrospectively.

\section{Patients and Methods}

Blood and serum samples from these individuals were submitted by an attending physician to the
Intracellular Pathogens Research Laboratory (IPRL) for attempted isolation or molecular detection of a Bartonella species. In most instances, these patients were tested because of a history of extensive arthropod or animal contact. Patient samples were tested as a component of an IRB approved study entitled: Detection of Bartonella Species in the Blood of People with Extensive Animal Contact (North Carolina State University Institutional Review Board, IRB\#s 4925-03 and 164-0805). None of the patients reported in this study have been described in previous manuscripts from our laboratory $[10,11,15]$. A standardized questionnaire was completed by each individual and included age, gender, animal and arthropod exposure, outdoor activity, travel, clinical symptoms, duration of illness and co-morbid conditions. The duration of illness varied substantially among individuals, as did prior diagnostic evaluations and previous treatments.

The authors used a previously described approach incorporating pre-enrichment culture of blood in Bartonella alpha Proteobacteria growth medium (BAPGM) followed by PCR targeting the 16S-23 S intergenic spacer (ITS) region and the rpoB gene $[10-13,16,17]$. Bacterial isolation, PCR amplification, and cloning were performed using previously described methods $[10,11,13,17]$. Sequences were aligned and compared with GenBank sequences using AlignX software (Vector NTI Suite 6.0, InforMax, Inc.) [17]. The BAPGM diagnostic platform consists of PCR following direct extraction of DNA from both blood and serum samples, PCR following enrichment culture for at least 7 days, and PCR following subculture onto a blood agar plate, if colony growth is visualized. To assess for potential laboratory contamination, an un-inoculated BAPGM culture flask was processed simultaneously and in an identical manner with each batch of patient blood and serum samples tested. Specifically, while establishing cultures using a batch of samples in a biosafety hood, the top was removed from the BAPGM un-inoculated control flask until all patient samples had been processed. Following the standard operating procedures in the Intracellular Pathogens Research Laboratory, sample preparation including BAPGM cultures and agar plate sub-inoculation, DNA extraction, PCR preparation and PCR amplification and analysis were performed in separate laboratory rooms to avoid culture as well as DNA contamination. In addition, negative and positive Bartonella DNA test control samples, consisting of bacteriafree blood DNA and DNA spiked with B. henselae genomic DNA at 0.5 genome copies per microliter, respectively, were used for each batch of DNA tested. To minimize chance contamination with whole bacteria, the laboratory does not use a BAPGM positive control and has never used a B. koehlerae PCR positive control. All 
PCR and BAPGM culture negative controls remained negative throughout the duration of the study.

Bartonella koehlerae, B. vinsonii subsp. berkhoffii, $B$. henselae and Rickettsia rickettsii antibodies were determined following traditional immunofluorescence antibody assay (IFA) practices $[10,11]$ with fluorescein conjugated goat anti-human IgG (Pierce Biotechnology, Rockford IL). Bartonella vinsonii subsp. berkhoffii genotype I (NCSU CO-1-93) and R. rickettsii (NCSU CO-108) originated from isolates made in our laboratory from natural canine infections. B. koehlerae (NCSU FO1-09) and B. henselae $\mathrm{H}-1$ (NCSU FO-93-23) were isolated from natural feline infections. Bartonella organisms were passed from agar grown cultures into AAE12 or DH82 cell cultures to obtain antigens as expressed when organisms are carried intracellularly in host blood. $R$. rickettsii cultures are maintained in cell lines due to the intracellular obligate growth requirements. Heavily infected cell cultures were spotted onto 30-well Teflon coated slides (Cel-Line/Thermo Scientific), air dried, acetone fixed and stored frozen. Serum samples were diluted in phosphate buffered saline (PBS) solution containing normal goat serum, Tween-20 and powdered nonfat dry milk to block non-specific antigen binding sites. All available patient sera were screened at dilutions of $1: 16$ to $1: 64$. All sera that were reactive at $1: 64$ were further tested with twofold dilutions out to 1:8192. To avoid confusion associated with possible non-specific binding found at low dilutions and to standardize with other laboratories such as the CDC, a cutoff titer of 1:64 was used to define a seroreactive titer. For preliminary testing purposes, $B$. koehlerae seroreactivity was assessed among six of the eight patients described in this study and using sera from 30 healthy people with minimal animal and arthropod exposure. A sufficient volume of serum was not available for 2 patients.

\section{Results}

Between 2005 and 2009 the IPRL performed 430 human blood cultures using the BAPGM enrichment platform, of which 8 individuals were found to be infected with $B$. koehlerae. There were four males and four females, ranging in age from 24 to 64 years, all of whom resided in the eastern United States (Table 1). Four of the eight individuals in this study were veterinarians, all of whom had historical animal contact for at least 10 years. Of the non-veterinarians, patients 1 and 7 reported animal contact of at least 10 years, patient 7 reported 6 to 10 years of animal contact, whereas patient 5 reported no recent animal contact. All veterinarians reported bites or scratches from cats, dogs and less frequently other animal species. Overall, fatigue, insomnia, joint pain, headache, memory loss, and muscle pain were the most frequently reported symptoms (Table 2).

For each patient, 16S-23 S ITS region PCR results were confirmed by DNA sequencing (Table 3). As the ITS region varies among Bartonella species and strains, sequence length ranged from 425 to 670 base pairs. Subsequently, the B. koehlerae rpoB gene was successfully amplified and sequenced (660 bp) from six of eight patient blood samples. In the context of the BAPGM platform, B. koehlerae was more often amplified directly from patient blood or serum samples, whereas the $B$. koehlerae PCR was positive only after enrichment culture for 7 or 14 days in BAPGM for three patients. Only one patient was PCR positive following both direct extraction and after enrichment culture. No subculture isolates were obtained from any patient in this study. To date, the cat isolate used as a source of IFA antigen in this study is the only $B$. koehlerae subculture isolate obtained in our laboratory after BAPGM enrichment culture.

By retrospective testing, $B$. koehlerae antibody titers ranged from $<1: 16$ to $1: 256$ in the six patients, for whom serum was available. Five of these six patients had titers of 1:64 or greater (Table 3). Bartonella koehlerae antibodies were not detected (titers $<1: 16$ ) in the 30 healthy control sera. Three patients had a 1:64 IFA titer to B. vinsonii subsp. berkhoffii and DNA of this subspecies was sequenced from these 3 patients, whereas one patient was PCR positive for $B$. vinsonii subsp. berkhoffii and $B$. henselae at different testing time points, but was not seroreactive to $B$. henselae. Due to the history of repeated seroreactivity to Rickettsia

Table 1 Age, gender, state of residence and duration of illness in eight patients infected with Bartonella koehlerae.

\begin{tabular}{cccll}
\hline Patient \# & Age & Gender & Occupation & State of Residence \\
\hline 1 & 24 & F & Manager, Retail Store & Pennsylvania \\
2 & 59 & F & Veterinarian & New Hampshire \\
3 & 61 & M & Veterinarian, Farmer & Florida \\
4 & 41 & F & Veterinarian & Virginia \\
5 & 64 & F & Homemaker & Maryland \\
6 & 27 & Artist & Maryland \\
7 & 48 & Mandscape Architect & North Carolina \\
8 & 33 & M & Veterinarian & North Carolina
\end{tabular}


Table 2 Symptoms reported by eight patients infected with Bartonella koehlerae.

\begin{tabular}{|c|c|c|c|c|c|c|c|c|}
\hline Symptoms & $\begin{array}{c}\text { Case } \\
1\end{array}$ & $\begin{array}{c}\text { Case } \\
2\end{array}$ & $\begin{array}{c}\text { Case } \\
3\end{array}$ & $\begin{array}{c}\text { Case } \\
4\end{array}$ & $\begin{array}{c}\text { Case } \\
5\end{array}$ & $\begin{array}{c}\text { Case } \\
6 \\
\end{array}$ & $\begin{array}{c}\text { Case } \\
7 \\
\end{array}$ & $\begin{array}{c}\text { Case } \\
8\end{array}$ \\
\hline Fatigue & $x$ & $x$ & $X$ & $x$ & & $x$ & $x$ & $x$ \\
\hline Insomnia & $x$ & $x$ & $x$ & $x$ & & $x$ & & $x$ \\
\hline joint pain & $x$ & $x$ & $x$ & $x$ & $x$ & & $x$ & $x$ \\
\hline Headache & $x$ & $x$ & $x$ & $x$ & & $x$ & & \\
\hline Memory loss & & $x$ & $x$ & $x$ & & $x$ & $x$ & \\
\hline Sleepiness & $x$ & & $x$ & $x$ & & & & \\
\hline Muscle pain & $x$ & $x$ & $x$ & $x$ & & $x$ & & $x$ \\
\hline Irritability & & $x$ & $x$ & $x$ & & $x$ & & \\
\hline Balance Problems & & $x$ & $x$ & $x$ & & $x$ & & \\
\hline Muscle weakness & $x$ & $x$ & $x$ & $x$ & & & & \\
\hline Blurred Vision & & $x$ & $x$ & & & $x$ & & \\
\hline $\begin{array}{l}\text { Loss of sensation } \\
\text { or numbness }\end{array}$ & $x$ & & $x$ & $x$ & $x$ & & & \\
\hline $\begin{array}{l}\text { Shortness of } \\
\text { breath }\end{array}$ & & & $x$ & $x$ & & & & $x$ \\
\hline Confusion & & $x$ & $x$ & & $x$ & & & \\
\hline Eye pain & & & $x$ & & & $x$ & & \\
\hline $\begin{array}{l}\text { Ventricular } \\
\text { arrhythmia } \\
\end{array}$ & & & & & & & & $x$ \\
\hline Depression & & & & & & $x$ & & $x$ \\
\hline Atrial Fibrillation & & & & & $x$ & & & \\
\hline $\begin{array}{l}\text { Incontinence, } \\
\text { urinary or bowel }\end{array}$ & & & $x$ & & & & & \\
\hline
\end{tabular}

rickettsii antigens in patient 7 , all patient sera were tested for potential cross reactivity to Rickettsia antigens. IFA titers to $R$. rickettsii ranging from 1:64 to1:128 were found in two patients (\#2 and \#7) (Table 3).

A brief medical history for each patient follows:

Patient 1 was examined by a rheumatologist in October 2008 due to recent onset of fatigue, headache, insomnia and bilateral hip pain. This patient reported daily contact with dogs, no direct contact with other animal species and minimal to no exposure to arthropods. Bartonella vinsonii subsp. berkhoffii was amplified and sequenced after direct extraction from a blood sample; however, growth was not obtained by BAPGM enrichment culture. She was not seroreactive to $B$. vinsonii subsp. berkhoffii or $B$. henselae antigens, but was retrospectively found to be seroreactive to $B$. koehlerae antigens (Table 3). Treatment with minocycline was accompanied by more frequent headaches and increased hip pain. She then received azithromycin with some decrease in headache frequency, but no change in hip pain. Following antibiotic administration, she was no longer B. koehlerae seroreactive; however, in February 2009, due to persistent fatigue, headache, hip joint and muscle pain, and newly acquired numbness and tingling sensations in the feet and hands, the patient was retested in the IPRL. B. koehlerae was amplified following direct extraction from blood, but bacterial DNA was not amplified from the enrichment or subcultures. Shortly after adding rifampin to the azithromycin in March 2009 she experienced a Jarisch-Herxheimer-like reaction with acutely worsening symptoms; however, after continuation of both antibiotics there was gradual improvement in energy level, decreased hip pain and less frequent headaches during the subsequent 2 months. In November 2009, two months after completing antibiotics, the woman reported decreased amplitude and frequency of hip pain.

Patient 2, a veterinarian with extensive companion animal contact, reported an eight-year history of illness, which was primarily characterized by muscle pain. Fibromyalgia was diagnosed in March 2003. After $B$. koehlerae was amplified and sequenced from a BAPGM enrichment culture in March 2009, the woman was treated with a 10-week course of rifampin and levofloxacin. Forty eight hours after starting antibiotics, she experienced a Jarisch-Herxheimer-like reaction, after which there was gradual, but definite clinical improvement, characterized by decreased stiffness and muscle pain, particularly in the morning and after exercise. Eight weeks after completing the course of antibiotics the patient reported resolution of muscle pain and metatarsophalangeal joint pain, which was reportedly swollen for ten years.

Patient 3, also a veterinarian, was chronically ill for 2 years. He had extensive contact with a spectrum of pet and production animals, also frequently participated in outdoor activities, including farming, hiking and hunting and had daily to weekly exposure to fleas, ticks, biting flies, and mosquitoes. His initial symptoms were most consistent with a severe, protracted flu-like illness, accompanied by fatigue and headaches. Afterwards, he developed numerous other symptoms which would wax and wane in severity, including shooting pain originating at the base of the skull and radiating over the head (Table 2). The most serious symptom described by this patient was frequent to near constant coughing resulting in production of large quantities of green sputum that induced choking and difficulty breathing. Based upon allergy test results, the patient was hyposensitized for nearly one year, without symptomatic improvement. Chronic obstructive pulmonary disease (COPD) was diagnosed, but no microbiological testing was performed. Approximately 6 months after development of bronchial signs the man developed sinusitis, which in March 2008 required surgical drainage. The respiratory illness had become so debilitating that continuing to work was a daily challenge and eventually the man avoided meetings and social gatherings due to the disruptive nature of his cough. B. koehlerae DNA was 
Table 3 Serological and PCR results for eight patients infected with Bartonella koehlerae.

\begin{tabular}{|c|c|c|c|c|c|c|c|}
\hline \multirow[t]{2}{*}{ Patient } & \multirow[t]{2}{*}{ Date } & \multicolumn{4}{|c|}{ IFA Reciprocal Titers } & \multicolumn{2}{|c|}{ PCR Results } \\
\hline & & B. koehlerae & B. henselae & $B v b I I$ & R. rickettsii & ITS PCR & rpoB PCR \\
\hline & $10-2008$ & 64 & $<16$ & $<16$ & $<16$ & $B v b^{*, a}$ & Neg \\
\hline 1 & $02-2009^{* *}$ & $<16$ & $<16$ & $<16$ & $<16$ & B. koehlerae ${ }^{a}$ & B.koehlerae $e^{a}$ \\
\hline 1 & $04-2009^{* *}$ & $<16$ & $<16$ & $<16$ & $<16$ & Bspp ${ }^{b}$ & Neg \\
\hline 2 & 03-2009 & $<16$ & $<16$ & 32 & 64 & B. koehlerae ${ }^{c}$ & B.koehlerae \\
\hline 2 & 04-2009 & NA & NA & NA & NA & Neg & Neg \\
\hline 2 & $07-2009^{* *}$ & $<16$ & $<16$ & $<16$ & 16 & B. koehlerae ${ }^{a}$ & Neg \\
\hline 2 & $10-2009^{* *}$ & $<16$ & $<16$ & $<16$ & $<16$ & Neg & Neg \\
\hline 3 & 03-2009 & 64 & $<16$ & 64 & $<16$ & B. koehlerae ${ }^{a}$ & B.koehlerae \\
\hline 3 & 04-2009 & NA & NA & NA & NA & B. koehlerae ${ }^{c}$ & Neg \\
\hline 3 & $05-2009^{* *}$ & 64 & $<16$ & 32 & $<16$ & Neg & $B \vee b^{*, a}$ \\
\hline 3 & $08-2009^{* *}$ & 256 & $<16$ & 16 & $<16$ & Neg & Neg \\
\hline 3 & $09-2009^{* *}$ & $<16$ & $<16$ & $<16$ & $<16$ & Neg & Neg \\
\hline 4 & 4-13-2009 & $<16$ & $<16$ & $<16$ & 32 & B. koehlerae & Neg \\
\hline 4 & $4-30-2009^{* *}$ & $<16$ & $<16$ & $<16$ & $<16$ & Neg & $B \vee b^{*, a}$ \\
\hline 4 & $6-02-2009^{* *}$ & $<16$ & $<16$ & 32 & $<16$ & Neg & Neg \\
\hline 4 & $6-09-2009^{* *}$ & $<16$ & $<16$ & 64 & 16 & Neg & Neg \\
\hline 4 & $6-30-2009^{* *}$ & 64 & $<16$ & 64 & $<16$ & Neg & Neg \\
\hline 5 & 05-2009 & NA & NA & NA & NA & B. koehlerae ${ }^{a}$ & B.koehlerae $e^{a}$ \\
\hline 6 & 05-2009 & NA & NA & NA & NA & B. koehlerae ${ }^{a}$ & B.koehlerae ${ }^{a}$ \\
\hline 7 & 04-2009 & 64 & $<16$ & 32 & 128 & B. koehlerae ${ }^{b}$ & Neg \\
\hline 7 & 06-2009 & 64 & $<16$ & 64 & 64 & Neg & $B \vee b^{*, a}$ \\
\hline 7 & 07-2009 & 64 & $<16$ & $<16$ & 64 & B. henselae ${ }^{a}$ & Neg \\
\hline 7 & $11-2009^{* *}$ & 256 & $<16$ & $<16$ & 16 & B. henselae ${ }^{a}$ & $\mathrm{Neg}$ \\
\hline 8 & 04-2009 & 64 & $<16$ & $<16$ & 16 & B. koehlerae & Neg \\
\hline 8 & 05-2009 & 64 & $<16$ & $<16$ & $<16$ & B. koehlerae ${ }^{a}$ & B.koehlerae \\
\hline 8 & 07-2009 & 64 & $<16$ & $<16$ & 32 & Neg & Neg \\
\hline 8 & 08-2009 & $<16$ & $<16$ & 32 & $<16$ & Neg & Neg \\
\hline
\end{tabular}

All ITS and rpoB PCR amplicons were sequenced to confirm genus and species identity.

${ }^{*} B v b=$ Bartonella vinsonii subsp. berkhoffii genotype II

Bspp $=$ amplicon generated with 16S-23 S ITS primers, but unable to obtain a DNA sequence.

** Denotes post-antibiotic testing. NA = Sample not available.

${ }^{\mathrm{a}}$ = amplicon sequenced from blood or serum

${ }^{\mathrm{b}}=$ amplicon sequenced from BAPGM enrichment blood culture

${ }^{c}=$ amplicons sequenced from both blood and BAPGM enrichment blood culture

amplified and sequenced following direct extraction from two blood samples obtained five weeks apart and for a third time from the initial BAPGM enrichment culture. After a 3-month course of doxycycline and rifampin, he experienced an increased energy level accompanied by substantial overall symptomatic improvement including resolution of the sinusitis, a nearly complete elimination of the cough and a notable decrease in right stifle joint crepitus, which he had previously attributed to old age. There was no relapse in symptoms during the eight month follow-up period.

Patient 4 developed retro-ocular headaches and pain in the elbow and shoulder in 1991, at the time of graduation from veterinary school. Within a year, pain also involved the hip joints and knees. Due to subsequent involvement of peripheral joints, accompanied by increasingly severe fatigue, the patient was cared for by a rheumatologist and various other medical specialists. 
Beginning in 2001, she developed a peripheral neuropathy. B. koehlerae DNA was amplified and sequenced from the initial BAPGM enrichment blood culture whereas $B$. vinsonii subsp. berkhoffii was amplified and sequenced directly from a post-treatment blood sample, obtained one month later. B. koehlerae DNA was concurrently amplified and sequenced from one of her pet cats. Following administration of doxycycline, Bartonella spp. DNA was not amplified from three subsequent blood culture attempts, but there was minimal change in symptoms. Beginning in August 2009, azithromycin and minocycline were prescribed for three months, during which time symptomatic improvement was reported. In March, 2010, the patient reported resolution in joint and muscle pain, peripheral neuropathy, neurocognitive abnormalities and proprioceptive deficits.

Patient 5 had a history of gastroparesis, paroxysmal atrial fibrillation and flutter that developed in December 2008 (status post ablation therapy), and a 2 year history of bilateral symmetric tingling in the first 3 toes of both feet with no loss of sensation, similar but milder neuropathy in her hands, and cognitive impairment, which she described as 'brain fog'. She had been treated medically for atrial fibrillation and osteoporosis. She reported minimal to no exposure to biting insects, no recent contact with animals, but had previously owned a dog. The patient had numerous small varicosities on the lower extremities, lower extremity bursitis; and tenderness in the right lateral quadriceps area. B. koehlerae was amplified directly from the blood sample but the BAPGM enrichment culture and subcultures were negative. Serum was not available for serological testing. In June 2009, azithromycin was administered for B. koehlerae infection. By July 2009 the woman reported improved cognition and short term memory, and increased energy. During the next 3 months, azithromycin was continued and the patient experienced increased symptoms of GI dysmotility, intermittent flu-like symptoms accompanied by intense chills without rigors and constant symmetric tingling of first 3 toes. Despite these symptoms, the "brain fog" and tingling in the hands were much improved, there was some improvement of neuropathy on left side, but the integrity of her short term memory fluctuated.

Patient 6 was diagnosed with idiopathic multi-focal retinitis by an ophthalmologist in August, 2008, which responded to high dose prednisone $(60 \mathrm{mg} /$ day $)$. The patient subsequently developed cardiac arrhythmias, characterized by extrasystoles and pauses, skeletal muscle twitching and spasms, and fatigue in November 2008. A fluorescence angiogram again documented retinal inflammation and prednisone therapy was re-instituted. In June 2009, the patient reported partial overall improvement in symptoms while taking prednisone. A rheumatoid factor assay was positive (21, reference range $0-14)$. A vasculopathy, with accompanying retinopathy, was diagnosed.

There was no history of tick bites, but the patient had rescued three feral cats, which he maintained as pets and had experienced cat scratches and bites. B. koehlerae was amplified directly from the blood sample, but the BAPGM enrichment culture and subculture were negative. The patient was treated with azithromycin and minocycline. When examined in December 2009, he reported overall symptomatic improvement, despite having not completed the entire course of antibiotics. Treatment with azithromycin and rifamipin for the next six months resulted in alleviation of symptoms, including no recurrence of visual floaters and light flashes.

Patient 7, who had daily dog contact and participated frequently in outdoor activities, reported recent onset of severe fatigue, difficulty remembering and joint pain and a history of tick exposure. During the past few years the patient developed a dietary allergy to beef and pork and fatigue, aches, "fogginess" that he had attributed to the aging process. Antibodies to Rickettsia rickettsii were previously detected by a commercial laboratory and antibodies were also detectable in the IPRL in three serum samples obtained at monthly intervals. This individual was consistently seroreactive to $B$. koehlerae antigens, intermittently seroreactive to $B$. vinsonii subsp. berkhoffii, but was never found to be seroreactive to $B$. henselae (Table 3). Bartonella koehlerae, B. henselae or $B$. vinsonii subsp. berkhoffii were amplified directly from four blood samples obtained over a seven-month time period. After a three-month course of doxycycline and rifampin, $B$. henselae DNA was again sequenced from an extracted blood sample in November 2009 and the $B$. koehlerae titer had increased from 1:64 to 1:256. During the months following antibiotic administration the patient reported gradual improvement in symptoms and by February 2010 he described a significant improvement in the "fogginess" and improved ability to remember and decreased joint pain.

Patient 8 The blood culture from Patient 8 was obtained due to the acute onset of ventricular tachyarrhythmia's that developed three weeks after coccygodynia surgery and during a professionally stressful period in this individual's life. Concurrently, this patient reported difficulty breathing, palpitations and anxiety. Other medical problems reported during the previous four years, included bilateral knee pain with patellar chondromalacia, documented by MRI, bilateral carpal tunnel syndrome and tinnitus involving the left ear. Although a veterinarian, with extensive prior animal contact, this individual had minimal occupational exposure during the 4-year period prior to PCR detection of $B$. koehlerae in the initial BAPGM enrichment blood 
culture. A blood sample obtained one month later also contained B. koehlerae DNA. As two subsequent blood cultures were $B$. koehlerae negative and because the patient was no longer $B$. koehlerae seroreactive, this individual elected to not be treated with antibiotics.

\section{Discussion}

Although biased by a study population consisting of individuals with extensive arthropod and animal exposure, the results of this study suggest that $B$. koehlerae bacteremia occurs more frequently ( 8 of 430 patients tested, $1.8 \%$ ) in immunocompetent patients than previously suspected. Also, as the duration of illness in these patients ranged from two months to eighteen years, $B$. koehlerae may induce a chronic intravascular infection, as recently proposed for $B$. vinsonii subsp. berkhoffii and $B$. henselae [18]. In addition, repeated amplification of $B$. koehlerae DNA from sequentially obtained blood samples from patients 2, 3, and 8 further supports the possibility of a persistent or relapsing pattern of bacteremia. Similar to two previous studies $[10,11]$, the mean age of this patient cohort was 45 years. The most frequent symptoms reported by these patients included fatigue, insomnia, joint pain, headache, memory loss, and muscle pain. Although non-specific, these symptoms are very similar to those previously reported in bacteremic patients that were infected with $B$. henselae, B. vinsonii subsp. berkhoffii, Candidatus B. melophagi or individuals co-infected with more than one Bartonella spp. $[10,11,15]$. Because recent studies describe very similar patterns of illness in immunocompetent patients $[10,11,15]$, prospective controlled studies are needed to critically assess whether there is an association between chronic intravascular infection with Bartonella spp. and specific disease entities. Importantly, sequential studies involving veterinarians and veterinary technicians, in conjunction with appropriate control groups, might allow for more rapid determination of the pathogenic potential of a growing number of zoonotic Bartonella spp. in immunocompetent individuals.

Our findings do not confirm that $B$. koehlerae was a cause or co-factor in the illnesses described in these patients or that the response to therapy was related to elimination of this bacteria. However, despite protracted medical histories, all seven patients treated with antibiotics reported substantial symptomatic improvement and in most instances B. koehlerae antibodies and DNA were no longer detected in post-treatment samples. Failure to consistently detect $B$. koehlerae bacteremia by repeat testing in patients 2 and 7 prior to antibiotic administration or in patient 8 who was never treated with antibiotics could relate to a relapsing pattern of bacteremia, as demonstrated following experimental inoculation of $B$. henselae and B. clarridgeiae into cats
[19]. Alternative explanations include limited sensitivity of the BAPGM platform for the detection of B. koehlerae, or immunological clearance of these bacteria from the vasculature. Although co-infection with more than one Bartonella sp. has been reported with increasing frequency in human patients $[10,11,18,20]$, the detection of $B$. vinsonii subsp. berkhoffii DNA in four of eight patients infected with $B$. koehlerae was an unexpected finding. Cats are considered the primary reservoir hosts for $B$. koehlerae, whereas dogs and wild canines are the primary reservoir hosts for $B$. vinsonii subsp. berkhoffii. Infection with $B$. vinsonii subsp. berkhoffii spanning an eighteen month time frame occurred in a cat with recurrent osteomyelitis [21]. Although the mode of $B$. vinsonii subsp. berkhoffii transmission could not be determined, patients 3 and 4 were companion animal veterinarians, who had frequent contact with both cats and dogs; whereas, patients 1 and 7 reported primarily dog contact.

When attempting to establish if a patient has been previously exposed to or is actively infected with $B$. koehlerae, serology, direct PCR from blood and serum and enrichment culture should be examined. Although IFA results reported in this study should be considered preliminary, five of six patients for whom serology was performed were $B$. koehlerae IFA seroreactive at titers of 1:64 to 1:256, whereas $B$. koehlerae antibodies were not detected in thirty healthy individuals. There was no serological crossreactivity to $B$. henselae antigens in these B. koehlerae infected patients. As reported for other $B$. henselae infected individuals $[10,11]$, patient 7 was never $B$. henselae seroreactive, despite sequencing $B$. henselae DNA at two temporally separated sample testing time points. In contrast to high antibody titers and serological crossreactivity reported in patients with Bartonella sp. endocarditis, these bacteremic patients had low antibody titers and minimal crossreactivity. Previous studies have suggested the possibility of serological cross reactivity between Bartonella and Rickettsia spp. antigens [22]. Patients 2 and 7 had $R$. rickettsii antibody titers ranging from $1: 64$ to $1: 128$, whereas patients 4 and 8 had very low titers (1:16 and 1:32 at different time points). There was no consistent pattern of cross reactivity among Bartonella and Rickettsia antigens in this study, therefore prior exposure to a Rickettsia sp. seems more likely than crossreactivity among Rickettsia and Bartonella sp. antigens. When specific pathogen free dogs were infected with $B$. vinsonii subsp. berkhoffii genotype I, there was no cross reactivity to $R$. rickettsii antigens by IFA testing [23].

The medical history of patient 3 , an older veterinarian, was unique in the context of severe, chronic bronchitis and sinusitis. The pulmonary disease was of such a severity that the man could only marginally function in 
routine work related activities. Atypical manifestations and more severe clinical presentations have been proposed to occur in older individuals infected with Bartonella spp. [24]. Although considered infrequent, pulmonary manifestations have been associated with $B$. henselae infections in patients with CSD [25]. In addition, $B$. henselae DNA was amplified and sequenced from nodular granulomatous pulmonary nodules that developed in a renal transplant recipient, who had extensive cat contact [26].

Patient 4 developed a peripheral neuropathy after a ten-year history of joint pain, whereas patients 1 and 6 reported tingling sensations in the hands or feet, which could be indicative of a sensory neuropathy. A distal axonal sensomotor polyneuropathy, vasculitis and Raynaud's phenomenon were reported in a 40 year-old man with a five year history of recurrent, painful toe ulcers [27]. Diagnosis of bartonellosis in that patient was supported by a $B$. henselae antibody titer of 1:1,024 and remission of the vasculitis and the polyneuropathy following treatment with erythromycin for three months followed by doxycycline for three weeks. Biopsy of the patient's sural nerve demonstrated an axonal neuropathy with a neurogenic muscular atrophy. The authors concluded that $B$. henselae infection should be considered in patients with vasculitis and polyneuropathic syndromes. It is also possible that $B$. koehlerae should also be among the differential diagnoses for patients with peripheral neuropathy.

Interestingly, three patients in this study reported atrial or ventricular tachyarrhythmias. Although an increasingly well recognized cause of endocarditis, the potential role of Bartonella spp. as a cause of myocarditis or arrhythmias has been incompletely studied [28]. Bartonella henselae has been implicated on the basis of molecular pathology as a cause of sudden death in Swedish orienteers [29]. In a more recent study from Switzerland, arrhythmogenic right ventricular cardiomyopathy (ARVC), an important cause of sudden death in young adults, was serologically associated with $B$. henselae antibodies [30]. These authors concluded that further studies in larger patient cohorts seem justified to investigate a possible causal link between chronic $B$. henselae and ARVC, in particular the sporadic (nonfamilial) form of the disease. Chronic active myocarditis requiring heart transplantation was also reported in a previously healthy young man after developing $B$. henselae-induced cat scratch disease [31]. In the context of intravascular pathogens, Bartonella species may prove to be unique, as these bacteria can induce intraerythrocytic infection, vascular endothelial infection and experimentally can achieve intracellular localization within monocytes, macrophages, dendritic cells and CD 34+ progenitor cells $[32,33]$. Assuming persistent infection coupled with a highly adapted ability to evade immune recognition by the host, these highly fastidious intravascular and intracellular bacteria could contribute to a spectrum of disease pathology over a protracted period of time [32].

On a daily basis, veterinarians are repeatedly exposed to a spectrum of animal species and to their associated arthropods. Unfortunately, due to the unknown duration of $B$. koehlerae infection in an immunocompetent person, the potential for repeated exposures over time and technical limitations associated with the molecular documentation of co-infection, the source and the timing of Bartonella infection in these individuals could not be accurately determined. Although veterinary professionals appear to be at a high risk of occupational exposure for Bartonella spp., numerous people in society have frequent or episodic exposure to animals and arthropod vectors. Increased attention to modes of transmission and risk factors for Bartonella spp. infections is warranted [34].

\section{Acknowledgements}

We wish to thank Tonya Lee for editorial support and the Division of Infectious Diseases, Duke University Medical Center for providing sera from the healthy control population. Supported in part by the state of North Carolina and grants from the American College of Veterinary Internal Medicine Foundation, the Kindy French Foundation and by Bayer Animal Health.

\section{Author details}

Intracellular Pathogens Research Laboratory and the Center for Comparative Medicine and Translational Research, College of Veterinary Medicine, North Carolina State University, Raleigh, NC, USA. ${ }^{2}$ Translational Medicine Group, Rockville, MD, USA.

\section{Authors' contributions}

EBB was involved in all aspects of this study, including generation of the initial draft of the manuscript; RGM and PEM performed all blood cultures, PCR, DNA sequencing and molecular data analyses, BRM was responsible for patient evaluations, medical record review and patient follow-up, $\mathrm{BCH}$ and $J M B$ were responsible for serological testing. All authors contributed to the content and approved the final manuscript.

\section{Competing interests}

In conjunction with Dr. Sushama Sontakke and North Carolina State University, Dr. Breitschwerdt holds U.S. Patent No. 7,115,385; Media and Methods for cultivation of microorganisms, which was issued October 3, 2006. He is the chief scientific officer for Galaxy Diagnostics, a newly formed company that provides diagnostic testing for the detection of Bartonella species infection in animals and in human patient samples. Dr. Ricardo Maggi performed all molecular microbiological testing reported in this study and is the Scientific Technical Advisor and Laboratory Director for Galaxy Dx.

Received: 3 August 2010 Accepted: 24 August 2010

Published: 24 August 2010

\section{References}

1. Doz S, Chi B, Horn E, Steigerwalt AG, Whitney AM, Brenner DJ: Bartonella koehlerae sp. nov., isolated from cats. J Clin Microbiol 1999, 37:1117-1122.

2. Koehler JE, Glaser CA, Tappero JW: Rochalimaea henselae infection: a new zoonosis with the domestic cat as reservoir. JAMA 1994, 271:531-535.

3. Yamamoto K, Chomel BB, Kasten RW, Hew CM, Weber DK, Lee WI, Droz S, Koehler JE: Experimental infection of domestic cats with Bartonella 
koehlerae and comparison of protein and DNA profiles with those of other Bartonella species infecting felines. J Clin Microbiol 2002, 40:466-467.

4. Rolain JM, Franc M, Davoust B, Raoult D: Molecular detection of Bartonella quintana, B. koehlerae, B. henselae, B. clarridgeiae, Rickettsia felis and Wolbachia pipientis in cat fleas, France. Emerg Infect Dis 2003, 9:338-342.

5. Marie JL, Fournier PE, Rolain JM, Briolant S, Davoust B, Raoult D: Molecular detection of Bartonella quintana, B. elizabethae, B. koehlerae, B. doshiae, B. taylorii, and Rickettsia felis in rodent fleas collected in Kabul, Afghanistan. Am J Trop Med 2006, 74:436-439.

6. Rolain JM, Fournier PE, Raoult $\mathrm{D}$, Bonerandi JJ: First isolation and detection by immunofluorescence assay of Bartonella koehlerae in erythrocytes from a French cat. J Clin Microbiol 2003, 41:4001-4002.

7. Avidor B, Graidy M, Efrat G, Leibowitz C, Shapira G, Schattner A, Zimhony O, Giladi M: Bartonella koehlerae, a new cat-associated agent of culturenegative human endocarditis. J Clin Microbiol 2004, 42:3462-3468.

8. Ohad DG, Morick D, Avidor B, Harrus S: Molecular detection of Bartonella henselae and Bartonella koehlerae from aortic valves of Boxer dogs with infective endocarditis. Vet Microbiol 2010, 141:182-185.

9. Chomel BB, Boulouis HJ, Maruyama S, Breitschwerdt EB: Bartonella spp. in pets and effect on human health. Emerg Infect Dis 2006, 12:389-394.

10. Breitschwerdt EB, Maggi RG, Duncan AW, Nicholson WL, Hegarty BC, Woods CW: Bartonella species in blood of immunocompetent persons with animal and arthropod contact. Emerg Infect Dis 2007, 13:938-941.

11. Breitschwerdt EB, Maggi RG, Nicholson WL, Cherry NA, Woods CW: Bartonella spp. bacteremia in patients with neurological and neurocognitive dysfunction. J Clin Microbiol 2008, 46:2856-2861.

12. Cherry NA, Diniz PPVP, Maggi RG, Hummel JB, Hardie EM, Behrend EN, Rozanski E, DeFrancesco TC, Cadenas MB, Breitschwerdt EB: Isolation or molecular detection of Bartonella henselae and Bartonella vinsonii subsp. berkhoffii from dogs with idiopathic cavitary effusions. J Vet Intern Med 2009, 23:186-189.

13. Duncan AW, Maggi RG, Breitschwerdt EB: A combined approach for the enhanced detection and isolation of Bartonella species in dog blood samples: Pre-enrichment culture followed by PCR and subculture onto agar plates. J Microbiol Meth 2007, 69:273-281.

14. Kosoy M, Morway C, Sheff KW, Bai Y, Colborn J, Chalcraft L, Dowell SF, Peruski LF, Maloney SA, Baggett H, Sutthirattana S, Sidhirat A, Maruyama S, Kabeya H, Chomel BB, Kasten R, Popov V, Robinson J, Kruglov A, Petersen LR: Bartonella tamiae sp. nov., a newly recognized pathogen isolated from three human patients from Thailand. J Clin Microbiol 2008, 46:772-775.

15. Maggi RG, Kosoy M, Mintzer M, Breitschwerdt EB: Isolation of Candidatus Bartonella melophagi from human blood. Emerg Infect Dis 2009, 15:66-68

16. Diniz PPVP, Wood M, Maggi RG, Sontakke S, Stepnik M, Breitschwerdt EB: Co-isolation of Bartonella henselae and Bartonella vinsonii subsp. berkhoffii from blood, joint and subcutaneous seroma fluids from two naturally infected dogs. Vet Microbiol 2009, 138:368-372.

17. Diniz PP, Billeter SA, Otranto D, De Caprariis D, Petanides T, Mylonakis ME, Koutinas AF, Breitschwerdt EB: Molecular documentation of Bartonella infection in dogs in Greece and Italy. J Clin Microbiol 2009, 47:1565-1567.

18. Breitschwerdt EB, Maggi RG, Farmer P, Mascarelli PE: Molecular evidence of perinatal transmission of Bartonella vinsonii subsp. berkhoffii and $B$. henselae to a child. J Clin Microbiol.

19. Kordick DL, Breitschwerdt EB: Relapsing bacteremia following blood transmission of Bartonella henselae in cats. Am J Vet Res 1997, 58:492-497.

20. Breitschwerdt EB, Maggi RG, Lantos PM, Woods CW, Hegarty BC, Bradley JM: Bartonella vinsonii subsp. berkhoffii and Bartonella henselae in a father and daughter with neurological disease. Parasit Vectors 2010, 3:29.

21. Varanat M, Travis A, Lee W, Maggi RG, Bissett SA, Linder KE, Breitschwerdt EB: Recurrent osteomyelitis in a cat due to infection with Bartonella vinsonii subsp berkhoffii genotype II. J Vet Intern Med 2009, 23:1273-1277.

22. Takeda N, Ishiwada N, Fukasawa C, Furuya Y, Tsuneoka H, Tsukahara M, Kohno Y: Pediatric pneumonia, pleural effusion, and pericarditis following cat scratch disease and serological cross-reactions among Bartonella henselae and Rickettsia japonica determined by indirect fluorescence. KantibodiesKansenshogaku Zasshi 2007, 81:206-209, Japanese.

23. Pappalardo BL, Correa MT, York CC, Peat CY, Breitschwerdt EB: Epidemiologic evaluation of the risk factors associated with exposure and seroreactivity to Bartonella vinsonii in dogs. Am J Vet Res 1997, 58:467-471.
24. Maman E, Bickels J, Ephros M, Paran D, Comaneshter D, Metzkor-Cotter E, Avidor B, Varon-Graidy M, Wientroub S, Giladi M: Musculoskeletal manifestations of cat scratch disease. Clin Infect Dis 2007, 45:1535-1540.

25. Margileth AM, Baehren DF: Chest-wall abscess due to cat-scratch disease (CSD) in an adult with antibodies to Bartonella clarridgeiae: case report and review of the thoracopulmonary manifestations of CSD. Clin Infect Dis 1998, 27:353-357.

26. Caniza MA, Granger DL, Wilson KH, Washington MK, Kordick DL, Frush DP, Blitchington RB: Bartonella henselae: etiology of pulmonary nodules in a patient with depressed cell-mediated immunity. Clin Infect Dis 1995, 20:1505-1511

27. Stockmeyer B, Schoerner C, Frangou P, Moriabadi T, Heuss D, Harrer T: Chronic vasculitis and polyneuropathy due to infection with Bartonella henselae. Infection 2007, 35:107-109.

28. Chomel BB, Kasten RW, Williams C, Wey AC, Henn JB, Maggi R, Carrasco S, Mazet J, Boulouis HJ, Maillard R, Breitschwerdt EB: Bartonella endocarditis: a pathology shared by animal reservoirs and patients. Ann N Y Acad Sci 2009, 1166:120-126.

29. Wesslen L, Ehrenborg C, Holmberg M, McGill S, Hjelm E, Lindquist O, Henriksen E, Rolf C, Larsson E, Friman G: Subacute Bartonella infection in Swedish orienteers succumbing to sudden unexpected cardiac death or having malignant arrhythmias. Scand J Infect Dis 2001, 33:429-438.

30. Fischer $H$, van der Loo AB, Shär GM, Zbinden R, Duru F, Brunckhorst $C$, Rousson V, Delacrétaz YE, Stuber T, Oechslin EN, Follath F, Jenni R: Serological evidence for the association of Bartonella henselae infection with arrhythmogenic right ventricular cardiomyopathy. Clin Cardiol 2008, 31:469-471.

31. Meininger GR, Nadasdy T, Hruban RH, Bollinger RC, Baughman KL, Hare JM: Chronic active myocarditis following acute Bartonella henselae infection (Cat scratch disease). Am J Surg Pathol 2001, 25:1211-1214.

32. Dehio C: Molecular and cellular basis of bartonella pathogenesis. Annu Rev Microbiol 2004, 58:365-390.

33. Mandle T, Einsele H, Schaller M, Neumann D, Vogel W, Autenrieth IB, Kempf VA: Infection of human CD34+ progenitor cells with Bartonella henselae results in intraerythrocytic presence of B. henselae. Blood 2005, 106:1215-1222.

34. Billeter SA, Levy MG, Chomel BB, Breitschwerdt EB: Vector transmission of Bartonella species with emphasis on the potential for tick transmission. Med Vet Entomol 2008, 22:1-15.

doi:10.1186/1756-3305-3-76

Cite this article as: Breitschwerdt et al:: PCR amplification of Bartonella koehlerae from human blood and enrichment blood cultures. Parasites \& Vectors 2010 3:76.

\section{Submit your next manuscript to BioMed Central and take full advantage of:}

- Convenient online submission

- Thorough peer review

- No space constraints or color figure charges

- Immediate publication on acceptance

- Inclusion in PubMed, CAS, Scopus and Google Scholar

- Research which is freely available for redistribution

Submit your manuscript at www.biomedcentral.com/submit
C Biomed Central 